Int. J. Electrochem. Sci., 12 (2017) 3950 - 3964

\title{
Selective Electrochemical Sensing of Dopamine and Ascorbic Acid Using Carbon Paste Electrode Modified with Cobalt Schiff Base Complex and a Surfactant
}

\author{
Naghmeh Hassanzadeh ${ }^{1}$,Hamid Reza Zare-Mehrjardi ${ }^{1,2, *}$ \\ ${ }^{1}$ Department of Chemistry, Payame Noor University, PO BOX 19395-3697 Tehran, Iran \\ ${ }^{2}$ Research Center of Environmental Chemistry, Payame Noor University, Ardakan, Yazd, Iran \\ *E-mail: $\underline{\text { hr_zare@pnu.ac.ir, zareanalyst@gmail.com, hr.zaremehr@yahoo.com }}$
}

doi: $10.20964 / 2017.05 .07$

Received: 8 October 2016 / Accepted: 26 February 2017 / Published: 12 April 2017

The complex N,N'-bis(3-ethoxysalicylidene)-4,5-dimetyl-1,2-phenylenediamino cobalt (II) chloride $(\mathrm{CoL})$ and 1-octanaminium,N,N,N-trioctyl bromide (as a cationic surfactant) are used for the preparation of the modified carbon paste electrode (CPE). By using the cyclic and differential pulse voltammetric methods (CV and DPV), the electrochemical behavior of dopamine (DA) and ascorbic acid (AA) is studied at the surface of the cobalt Schiff base complex-modified CPE containing different percents of cationic surfactant. The anodic peak potential of AA is decreased, by introducing the cobalt Schiff base complex in the matrix of the modified CPE. On the other hand, in $\mathrm{pH} 4.0$ buffer solutions, in which all studies are carried out, DA exists as the positively charged species whereas AA is mainly as the neutral form. Therefore, there is low electrostatic attraction between the neutral form of AA and cationic surfactant. However by application of cobalt Schiff base complex-modified CPE containing cationic surfactant, the anodic overpotential for DA oxidation is increased. This effect is the result of the electrostatic repulsive interaction between the cationic form of DA and cationic surfactant at the surface of the modified electrode and results in very good resolution between the anodic peaks of $\mathrm{DA}$ and AA. The effects of the buffered solution $\mathrm{pH}$ and potential sweep rate on the response of the electrode, toward AA and DA, and the peak resolution is thoroughly studied by CV and DPV. Using the modified electrode, the best peak separation for these compounds is obtained in solutions with $\mathrm{pH}$ 4.0. These results reveal that the modified CPE has an effective electrocatalytic activity for the electrochemical oxidation of DA and AA and a peak resolution nearly $440 \mathrm{mV}$ is obtained for two compounds. The very easy preparation and surface regeneration of the modified electrode together with the efficient electrocatalytic property and very good peak resolution and reproducibility of the voltammetric responses designate the modified CPE appropriate for simultaneous voltammetric determination of DA and AA.

Keywords: cobalt Schiff base complex, cationic surfactant, modified carbon paste electrode 


\section{FULL TEXT}

(C) 2017 The Authors. Published by ESG (www.electrochemsci.org). This article is an open access article distributed under the terms and conditions of the Creative Commons Attribution license (http://creativecommons.org/licenses/by/4.0/). 